\title{
Sleep Nasopharyngoscopy in Children with Snoring/Sleep Disordered Breathing: Purpose and Validity
}

\author{
Maleka Ramji • Manisha Witmans • \\ Dominic Cave $\cdot$ Hamdy El-Hakim
}

Published online: 8 January 2013

(c) Springer Science+Business Media New York 2013

\begin{abstract}
The purpose of this work is to review the basis and validity of sleep nasopharyngoscopy (SNP) in children with sleep disordered breathing (SDB). We performed an English literature review of the effectiveness of modalities guiding surgical treatment, and the utility of SNP. Scant literature is available on SNP. Only two validation studies exist on adults, and none on children. Multiple-level pathology has been identified by several studies in children, but credible epidemiological work does not exist. Other modalities were studied but methodology flaws are substantial. In the absence of alternative validated diagnostic modalities, sleep endoscopy is emerging to identify surgical targets. This has mechanistic support, but as yet has not
\end{abstract}

M. Ramji · H. El-Hakim ( $\square)$

Pediatric Otolaryngology, Division of Pediatric Surgery,

Division of Otolaryngology, The Stollery Children's Hospital, University of Alberta Hospitals, 2C3.57 Walter MacKenzie

Centre, Edmonton, AB T6G 2R7, Canada

e-mail: hamdy.elhakim@albertahealthservices.ca

M. Ramji

e-mail: maleka@ualberta.ca

M. Ramji

453 Village Square, Sherwood Park, AB T8A 4K2, Canada

M. Witmans

Division of Pediatric Pulmonology, Department of Pediatrics,

The Stollery Children's Hospital, University of Alberta

Hospitals, Suite 201, 501 Bethel Drive, Sherwood Park,

AB T8H 0N2, Canada

e-mail: manishawitmans@gmail.com

D. Cave

Division of Pediatric Anesthesiology, The Stollery Children's

Hospital, University of Alberta Hospitals, 1C1.04 Walter C.

Mackenzie Centre, 8440-112 Street, Edmonton, AB T6G 2B7, Canada

e-mail: dcave@ualberta.ca been accompanied by the description of a valid scheme in evaluating the upper airway.

Keywords Sleep · Endoscopy · Obstructive sleep apnea · Children · Diagnosis · Surgery · Adenotonsillectomy ·

Cine magnetic resonance imaging $\cdot \mathrm{X}$-ray

\section{Introduction}

The spectrum of sleep disordered breathing (SDB) is well recognized, and its most severe form is obstructive sleep apnea syndrome (OSAS) [1]. The pathological mechanisms include one or more of genetic factors, structural narrowing of the airway, altered neuro-motor tone, impaired sensation, and inflammation [2••]. The prevalence estimates of OSAS in children, based on questionnaires, range from 1.2 to $5.7 \%[3 \cdot \bullet, 4 \cdot \bullet]$. This suggests that the full spectrum is a common condition. Untreated SDB is associated with significant sequelae across multiple domains, including increased health care utilization [5], cardiovascular dysfunction [6, 7], neuro-cognitive and neurobehavioral abnormalities, including attention deficit disorder [8-13].

The literature proposes that early identification and treatment may reverse these sequelae [14], and improve the child's long-term outcomes (improved behaviour, attention and social interactions) $[15 \cdot \bullet]$. Whereas medical management plays a definite role, surgery remains the primary therapy, usually in the form of adenoidectomy, tonsillectomy, or both (T\&A).

Overnight polysomnography (PSG) identifies and quantifies the abnormalities in sleep and/or gas exchange associated with SDB, and is superior to a multitude of other

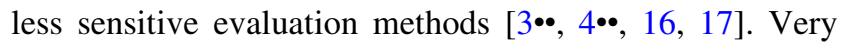
recently, a clinical practice guideline reinforced its status 
as a reference standard, and advocated its use as a preoperative investigation [16]. Unfortunately, PSG is unable to identify the level of obstruction or the optimal surgical target(s).

\section{Results of T\&A and Implications}

Although T\&A is currently the most common initial treatment for pediatric OSA, inconsistent success in curing OSA has led to a call for more research. [18]. A retrospective study conducted at six pediatric sleep centers in the United States and two in Europe demonstrated that T\&A surgery did result in improvements of sleep-indices. A complete resolution of OSA, however, was only achieved in $27.2 \%$ of cases. Residual disease was noted to be more prevalent in older, asthmatic and obese children [19].

This impression had been also expressed in a recently updated systematic review of the literature, which demonstrated that the success of T\&A in children was $66.3 \%$, when 'cure' was established on a case-by-case basis. When the threshold for 'cure' was determined as an apneahypopnea index (AHI) of $<1$, the success rate was $59.8 \%$ $[20 \bullet$. These results are likely a reflection that adenotonsillar enlargement is among several causes of narrowing of the upper airway, which is, in turn, one of several pathophysiological mechanisms that mediate SDB. At this point in time, there are no epidemiological data on the exact nature and prevalence of other causes of narrowing of the upper airway (especially in otherwise healthy children). We are also unable to consistently identify the children who have mechanisms of SDB that are, by nature, untreatable by T\&A, such as neuromuscular incoordination and sensory impairment of the upper airway [21].

\section{Literature on Non-endoscopic Modalities for Demonstration of Surgical Targets}

Traditionally, clinical examination in the outpatient setting has been the bedrock for the decision to operate. But a systematic review, conducted by Breitzke et al. [22] found that clinical evaluation alone, accounting for patient history and physical exam, was insufficient for the diagnosis of $\mathrm{SDB}$, and quoted a modest positive predictive value of only $55.8 \%$. A more recent review found again no association between subjective grading of tonsil size and the diagnosis of SDB [23]. It therefore appears that clinical examination and history do not reliably diagnose the condition, and that there were no attempts to define surgical targets, or predict surgical success in a systematic fashion.

Some radiological modalities have been investigated alone, and also in conjunction with clinical examination.
These include plain X-rays, and magnetic resonance imaging (MRI). A small study demonstrated that tonsil size on plain X-rays correlated with PSG findings, but the results have not been replicated [24]. Another study reported that the presence of abnormal AHI correlated with adenoidal hypertrophy as measured on a lateral X-ray of the nasopharynx, but not with tonsillar size on examination [25]. These findings suggest that while overall upper airway obstruction may predict abnormal AHI, the method does not delineate specific surgical target or targets.

Another target for this research has been children who failed T\&A. A group from Cincinnati examined cine magnetic resonance imaging (c-MRI) in these patients [26]. This group demonstrated statistically significant differences in upper airway measurements and collapse between 16 children with OSA and 16 children without. Children with Down syndrome who had already undergone T\&A and had residual SDB were also examined [27]. These children exhibited relative macroglossia (74 \%); glossoptosis (63\%), recurrent and enlarged adenoids (63\%); enlarged lingual tonsils (30\%), and hypopharyngeal collapse $(22 \%)$. These findings have been reproduced at the same centre in another Down syndrome children cohort [28]. Another study examined MRI measurements in 28 children and young adults, (mean age 11.2 years) who had undergone genioglossus advancement surgery for sleep apnea, to identify potential predictors of success [29]. Relative tongue size and small adenoid size were the two predictors of successful treatment.

There is little doubt that c-MRI provides for excellent demonstration of collapses and obstruction of the airway, in addition to digitized volumetric assessment, delineation of soft tissues and parapharyngeal structures, and does not expose the child to radiation. However, it still requires anaesthesia or sedation, is expensive and is not readily accessible. Together with the methodological limitations of the studies (mainly selection bias and small numbers), these factors limit its general applicability. Other non-invasive methods, to localize the level of airway obstruction or collapse have been examined. Anterior rhinomanometry showed good positive and negative predictive values (97, $86 \%$ respectively) in one study in children with SDB [30]. Acoustic pharyngometry [31], also shows reduced minimum cross-sectional area in children with SDB compared to those without. Unfortunately, neither modality can identify a surgical target with precision, and have not been adequately evaluated for implementation into regular clinical practice.

\section{Current Literature on Sleep Endoscopy in Adult Patients}

We conducted a comprehensive literature search on sleep endoscopy in the management of SDB. Medline, PubMed, 
ACP Journal Club and Cochrane Databases were all referenced. MeSH words used included 'endoscopy', 'sleep disordered breathing' and 'validation studies'; key words used included nasoendoscopy; and possible limits placed included pediatric and human studies. Using varying combinations, a total of 520 citations resulted. Finally, we used seventeen references for the purpose of this report.

Hessel and deVries conducted endoscopy on 380 adult patients diagnosed with unacceptable snoring (sic) to determine the sites of obstruction along the upper airway. They detected single level obstruction in only $35 \%$ of the patients, while $65 \%$ had multiple-levels [32]. The authors felt that the advantages for using endoscopy are detecting the multi-level nature of the pathology, demonstration of the surgical target and the assessment of the problem in a state similar to what is actually experienced by the patient and/or partner. Sadaoka et al. [33] reported that the findings of endoscopy correlated with non-REM PSG. However, only a small number of studies proposed a structured scoring scheme, or a validation for the investigation. Pringle and Croft [34] proposed a five-grade system after scoping 100 adult patients. These ranged from simple palatal level snoring, to single palatal level obstruction, then palatal level obstruction with intermittent orohypopharyngeal involvement, then consistent multi-segment involvement, and finally tongue-base level obstruction. The system was not validated.

Worthy of mention are two later prospective studies, conducted by the same research team who coined the acronym DISE (drug induced sleep endoscopy). These projects were test-retest reliability and inter-rater reliability of DISE in adults with SDB [33, 34]. The first study included thirty-two patients undergo two separate examinations using a structured scoring system, conducted and interpreted by two experienced sleep surgeons (only one of whom was blinded). Evaluation of findings was organized into three analyses. These involved a global assessment of obstruction at two levels: the palate and hypopharynx, the extent of obstruction at each site, and finally to determine which specific structure contributed primarily to the obstruction at the level of the palate and hypopharynx. The authors acknowledged the limitations of the exercise, particularly the assumption that only two potential independent regions of pathology existed (palate and hypopharynx). Using intra-class correlation coefficient analogs, the test-retest reliability of the scoring in this study was found moderate to substantial. Other limitations were the small sample size (yielding wide confidence intervals), a population with little heterogeneity (making it difficult to assess the true value of the classification system), and that the assessors were both experienced sleep surgeons, thereby limiting the generalizability of the results [35•*].
In the subsequent study assessing inter-rater reliability, an identical classification method was used. The same observers participated in the study; one was blinded, while the other was not. The agreement between reviewer ratings was calculated using Cohen kappa and weighted kappa statistics. The authors were content that agreement of the observers was substantial for global assessment of obstruction, and moderate for the degree of the obstruction. Also they concluded that the agreement was substantial on evaluating the primary structures contributing for the obstruction, than for individual structures. Although the sample size was larger than the earlier study, the disadvantages of using experienced observers, and limited variation in the sample persisted [36••].

\section{Pediatric Literature}

The articles on SNP trials in children are even more limited. A very early description of SNP investigation was provided by Sher et al. in 1986 . These authors explicitly limited their work to syndromic children, and the research was later extrapolated specifically to Pierre-Robin patients. Although their work included a classification of the findings into four types of upper airway findings (two types of glossoptosis, and two types of pharyngeal collapse), their methodology was never subjected to validation [37•].

Croft et al. [38] described the findings of endoscopy in a small series ( $\mathrm{n}$ of 15; amongst them six Down's children and one Pierre-Robin sequence) of children with proven sleep apnea. In their work, they did acknowledge the initial descriptions by Borowiecki et al. [39] who conducted a fibreoptic study of the pharyngeal airways during sleep in patients with hypersomnia, and OSAS.

Later on, Contencin et al. reported on 17 children (in almost 12 months) who had either symptoms of OSAS and/or abnormal PSG ( $n$ of 5), but with no clear site of obstruction on awake clinical examination. They used nitrous oxide and halothane for anesthesia, prior to which they examined the patients awake with topical anesthesia. Only nine had pure adenotonsillar obstruction judged to be the cause of the obstruction. In their discussion, they claimed that the investigation could spare the resource of the PSG. They contended that on awake clinical examination, the role of the adenoid and tonsillar hypertrophy in the generation and maintenance of the OSA symptoms cannot be always predicted. The authors postulated as well the potential role of a proximal, seemingly innocuous, point of obstruction producing downstream progressive, more severe obstruction, and cautioned against false positive findings [40].

Myatt and Beckenham, investigated and treated 20 children with AHI $>30 / \mathrm{h}$, largely with complex syndromic 
or dysmorphic diagnoses, amongst whom some had previous T\&A. Halothane and oxygen were used throughout the examination [41••]. This paper described four levels of findings: level 1 (velopharyngeal/adenoidal obstruction), level 2 (tonsillar/lateral pharyngeal wall), level 3 (tongue base obstruction) and level 4 (supraglottic obstruction) $[41 \bullet \bullet$. Unfortunately no validation exercise was undertaken, and the population was a complex one, leaving out otherwise healthy patients. Finally, a retrospective study conducted by Goldberg et al. identified on endoscopy both fixed and dynamic causes of upper airway obstruction. Forty-nine patients diagnosed with OSA (by PSG) were examined. Of these, 38 had endoscopy to identify potential upper airway obstruction while 11 were evaluated for other indications. Endoscopy identified areas of multiple obstructions along the upper airway: adenoidal hypertrophy (64\%), tonsillar hypertrophy (31\%), laryngomalacia (44\%) and pharyngeal wall collapse (41\%) [18].

Thevasagayam et al. [42•], reported a $3.9 \%$ prevalence rate of laryngomalacia in a retrospective consecutive case series of 358 children with SDB who were all examined endoscopically. Although the authors did not propose a validated structured scoring scheme, the message was mainly about documenting one type of airway pathology which clinician do not search for in that group of patients.

In 2012, Fung et al. [43*•] published a case-control retrospective study describing a structured system of SNP. They age-, gender-, and weight- (body mass index percentile) matched 23 Down syndrome children with counterparts who still presented with SDB. The endoscopic findings were reported as either obstructive, collapse or mixed findings. They demonstrated that pharyngeal collapse was statistically more prevalent in the Down syndrome group, along with tongue collapse. However, adenoid enlargement was significantly more in the controls. The argument to use such a scheme was primarily avoidance of unnecessary surgery and demonstration of a different mechanism of SDB. Unfortunately, the system is as yet to be validated, and the diagnosis of SDB was based on overnight pulse oximetry grading.

Truong et al. [44•] studied perhaps the largest group of patients to date ( $\mathrm{n}$ of 80 ). Total intravenous agents were used to induce sleep (propofol and remifentanil). Four levels of the airway were assessed (velum, oropharynx, tongue base, and supraglottis), but were not rated. There was not stratified display of the distribution of findings, rather the paper concentrated on the response to surgery (i.e., improvement in AHI). The authors were content that endoscopy-directed surgery improved all AHI scores for both the children who underwent T\&A previously and those who did not. This stratification probably diluted the sample size, but it was a re-emphasis on the relevance of surgical targets other than tonsils and adenoids.
Very recently, a retrospective study [45] used SNP in children with persistent SDB (AHI $>1 / \mathrm{h}$ ) after T\&A. Thirteen children, including five Down syndrome patients and three with cerebral palsy, were studied. The endoscopic findings were documented according to a modification of a four-level previously described method (VOTE; velum, oropharyngeal lateral walls, tongue base, and/or epiglottis) [46]. The results showed that multi-level obstruction, including tongue-based obstruction, inferior turbinate hypertrophy, and adenoidal re-growth, were contributing factors [28]. The series represented another highly select group, a small sample, and used a nonvalidated score in children.

The relatively scant published literature, therefore, has largely limited the use of SNP to small numbers of a highly select group of children, either because their SDB was mediated via a unique characteristic (syndromic or dysmorphic), or due to failure of an initial T\&A. Currently, we do not have established technical protocols, nor do we have epidemiologic data on possible locations of upper airway obstruction, other than tonsil and adenoid enlargement. Lastly, there is no consensus on how should we grade the information documented (i.e., score) or its significance in the overall management.

\section{Drug Related Considerations}

SNP is proposed as a valuable tool to assess airway collapse and obstruction. To be useful in clinical decision making, however, it must meet certain requirements. Findings under this technique should correlate with SDB, and the drugs used should provide reliable and reproducible conditions. Most importantly, the airway conditions in the drug-induced state should mimic those of natural sleep as closely as possible, and recent work has tried to address these issues.

The choice of possible sedative agents is wide. Initially various halogenated anesthetic agents were the most common choice. Then propofol infusions were implemented, as well as ketamine and benzodiazepines. More recently, dexemetomidine has been suggested, as it appears to more closely replicate REM sleep.

The choice of the agent should rest on the best available evidence for producing sleep-like airway conditions, at a reproducible assessment point. During sleep there is a very significant reduction in the airway tone mediated by a reduction in genioglossus stimulation [2••]. An ideal agent would produce this reduction in tone at a reliable depth of anesthesia.

Can sedated assessment be compared to normal sleep? Some differences exist in the available literature. Rapid eye movement (REM) sleep is reportedly abolished under 
propofol sedation, and slow-wave sleep is increased. This could lead to underestimation of the severity of SDB in children, as this tends to be more REM-related. Thus, the change in sleep architecture could have important implications for the use of propofol [47•]. However, Rabelo et al. [47•] also documented that adults undergoing polysomnography (PSG) with or without propofol sedation had very similar apnea hypopnea index (AHI) in both tests, suggesting a very similar impact on the airway of natural sleep and propofol sedation. Eastwood et al. [48] have also demonstrated that airway obstruction under isoflurane general anesthesia predicted SDB with AHI $>10$ by PSG.

The agent supported with the best evidence at this time is propofol. A number of studies have looked at its effect on genioglossus activity, and on critical obstruction pressures $[49,50 \bullet, 51]$. Pharyngeal critical closing pressure (Pcrit) measurement are used to quantify airway collapsibility by assessing the amount of applied negative pressure an airway can tolerate before obstruction occurs, or alternatively the amount of positive pressure required to prevent obstruction. The higher the Pcrit, the more collapsible the airway. Propofol has been found to consistently reduce genioglossus activity in a dose-dependent manner [51]. Critical pressure, however, only changes by a small amount initially as the dose increases. Then it undergoes an acute increase that is simultaneous with clinical unresponsiveness. Bispectral index (BIS) recordings at this time also drop suddenly, as would be expected when consciousness is lost [50•0]. This is similar to what is seen in natural sleep. Sleep is a complex process, but some elements are known to be present. For sleep to occur, the ventrolateral preoptic nucleus needs to be active, which is achieved through GABA receptor stimulation; propofol is known to act through these receptors [50•0. During sleep the hypoglossal nucleus becomes less active, and this results in a reduced activity of the genioglossus muscle, thus reducing airway tone. This hypoglossal nucleus down regulation is partially mediated by GABA receptors, which again are acted on by propofol.

Therefore, under propofol sedation, clinical findings are very similar to the PSG results found under normal sleep, and a biological mechanism by which the drug can act on the centers responsible for changes in airway tone during normal sleep. This, together with findings showing expected pathophysiologic airway changes at the onset of clinical sedation, make it a good candidate as an agent for SNP.

Midazolam has been directly compared to propofol in volunteers and was found to have a similar propensity for obstruction [49]. It too works through GABA activity, but has a longer onset and offset time. Benzodiazepines may also have a greater impact on depth of respiration, adding another confounder. Ketamine has been shown to increase genioglossus activity and increase secretions, and therefore may not be an appropriate agent for SNP assessment [52].

Halogenated inhalational anesthetic agents, like halothane, isoflurane and more recently sevoflurane, have also been employed [53, 54]. All these agents do suppress genioglossus activity to varying degrees, however the suppression is seen even at subanesthetic concentrations which makes it difficult to determine the optimal point at which the findings are most likely to reflect those of normal sleep. This may be a result of the presence of both glycine receptors and GABA receptors in the hypoglossal nucleus. Halogenated agents are known to act on both receptors and this may explain the earlier and less predictable depression of genioglossus activity.

Dexemetomidine produces a sedated state that allows easy rousing when given by infusion. It has been proposed as a possible agent for SNP in children. A study examining the impact of dexemetomidine versus propofol on cineMRI findings, found that children with dexemetomidine required fewer airway interventions compared to those with propofol [55]. Children with severe obstructive disease on sleep study (obstructive index $>10$ events/h) required an artificial airway more than $50 \%$ of the time in the propofol group versus $7 \%$ of the time in the dexemetomidine group. This, however, suggests that dexemetomidine did not mimic the sleep study findings as clearly as the propofol did. This could be explained if dexemetomidine sedation is a result of alpha-2 receptor activity, not GABA activity. It is therefore less likely to influence the hypoglossal nucleus in the same way. More work needs to be done to establish if dexemetomidine is a suitable agent for SNP assessment.

The current literature therefore suggests that a clinical target of loss of responsiveness can be used to achieve airway conditions that mimic findings seen in normal sleep using either propofol, or midazolam infusion, with propofol having a shorter offset time and a demonstrated threshold level for airway changes that closely matches clinical loss of responsiveness.

Research on SNP thus far has described the prevalence of laryngomalacia amongst children presenting primarily with SDB [42•], providing some unique epidemiological data, and offering one alternative to T\&A or a 'plan B' after its failure. Other authors provided some support to this notion [56]. Similarly, lingual tonsil enlargement has been identified as a potential surgical target [57]. Our unpublished data indicates that only one-fifth of children with SDB has single level abnormality.

SNP may allow characterization of the upper airway of different risk groups. Down syndrome patients have been shown to exhibit more collapse than obstruction [55]. Since this risk group (and others, like obese children) [58], are at increased risk of morbidity peri-operatively, these results 
can have far reaching implications. A further step would be to demonstrate if collapse may predict failure of surgical treatment as was recently claimed in an adult-based study [59•].

\section{Conclusions}

T\&A surgery for SDB in children is currently failing to cure many patients. The literature suggests that these children may have problems at multiple levels, and by multiple mechanisms. The risk of T\&A surgery requires that we apply this therapy only to those children who will benefit. SNP allows dynamic assessment of the airway, in a non-invasive manner. It may help select the most appropriate surgery for each individual child, avoid unnecessary surgery, and plan contingency treatment in cases of residual or recurrent symptoms. Along with other variables significant for SDB, SNP findings may be able to help predict failures of surgical therapy. This assertion will require further research.

The method proposed to direct surgical therapy should be supported by adequate level of evidence on reproducibility, accessibility, cost effectiveness, real-time representation, and accurate identification of all levels of pathology, and for a representative population and subpopulations. The method should also correlate with the presence of SDB, and prove predictive of the result of surgical intervention, along with other relevant patient variables. For decades, endoscopy has been routinely used to evaluate the upper and lower airway, and plan for treatment of various conditions. It is quite logical, then, that SDB should not be an exception to that rule. The established advantages include availability and familiarity, including a real time assessment of the airway under a simulated set of circumstances.

SNP, however, will never be a replacement for reference standard sleep studies, nor do we predict that it can be a sole determinant of treatment irrespective of the other patient and disease-related variables.

Disclosure No potential conflicts of interest relevant to this article were reported.

\section{References}

Papers of particular interest, published recently, have been highlighted as:

- Of importance

•• Of major importance

1. American Thoracic Society. Standards and indications for cardiopulmonary sleep studies in children. Am J Respir Crit Care Med. 1996;153(2):886-878.
2. • White DP. Pathogenesis of obstructive sleep apnea. Am J Respir Crit Care Med. 2005;172(11):1363-1370. Important information on pathogenesis and essential reading on sleep pathophysiology

3. - Marcus CL, Brooks LJ, Draper KJ, Gozal D, Halbower AC, Jones J, et al. Diagnosis and management of childhood obstructive sleep apnea syndrome. Pediatrics 2012;130(3):e714-755. Guideline

4. • Marcus CL, Brooks LJ, Draper KA, Gozal D, Halbower AC, Jones J, et al. Diagnosis and management of childhood obstructive sleep apnea syndrome. Pediatrics 2012;130(3):576-584. Guideline

5. Tarasiuk A, Simon T, Tal A, Reuvenu H. Adenotonsillectomy in children with obstructive sleep apnea syndrome reduces health care utilization. Pediatrics. 2004;113:351.

6. Amin RS, Kimball TR, Bean JA, Jeffries JL, Willging JP, Cotton RT, et al. Left ventricular hypertrophy and abnormal ventricular geometry in children and adolescents with obstructive sleep apnea. Am J Respir Crit Care Med. 2002;165(10):1395-9.

7. Amin RS, Kimball TR, Kalra M, Jeffries JL, Carroll JL, Bean JA, et al. Left ventricular function in children with sleep-disordered breathing. Am J Cardiol. 2005;95(6):801-4.

8. Key AP, Molfese DL, O'Brien L, Daniels SR. Sleep-disordered breathing affects auditory processing in 5-7-year-old children: evidence from brain recordings. Dev Neuropsychol. 2009;34:615-28.

9. Owens JA. Neurocognitive and behavioral impact of sleep disordered breathing in children. Pediatr Pulmonol. 2009;44:414-22.

10. Gozal D. Sleep-disordered breathing and school performance in children. Pediatrics. 1998;102:61-620.

11. Montgomery-Downs HE, O'Brien LM, Holbrook CR, Gozal D. Snoring and sleep-disordered breathing in young children: subjective and objective correlates. Sleep. 2004;27:87-94.

12. Gozal D, Crabtree VM, Sans Capdevila O, Witcher LA, Kheirandish-Gozal L. C-reactive protein, obstructive sleep apnea, and cognitive dysfunction in school-aged children. Am J Respir Crit Care Med. 2007;176:188-93.

13. Chervin RD, Dillon JE, Bassetti C, Ganoczy DA, Pituch KJ. Symptoms of sleep disorders, inattention, and hyperactivity in children. Sleep. 1997;20:1185-92.

14. Koc S, Aytekin M, Kalay N, Ozcetin M, Burucu T, Ozbek K, et al. The effect of adenotonsillectomy on right ventricle function and pulmonary artery pressure in children with adenotonsillar hypertrophy. Int J Pediatr Otorhinolaryngol. 2012;76(1):45-8.

15. $\bullet$ Beebe DW. Neurobehavioral morbidity associated with disordered breathing during sleep in children: a comprehensive review. Sleep 2006;29(9):1115-1134. Review on impact of SDB on behaviour

16. Roland PS, Rosenfeld RM, Brooks LJ, Friedman NR, Jones J, Kim TW, et al. Clinical practice guideline: Polysomnography for sleep-disordered breathing prior to tonsillectomy in children. Otolaryngol Head Neck Surg. 2011;145(Suppl 1):S1-15.

17. Aurora RN, Zak RS, Karippot A, Lamm CI, Morgenthaler TI, Auerbach SH, et al. Practice parameters for the respiratory indications for polysomnography in children. Sleep Am Acad Sleep Med. 2011;34(3):379-88.

18. Goldberg S, Shatz A, Picard E, Wexler I, Schwartz S, Swed E, et al. Endoscopic findings in children with obstructive sleep apnea: Effects of age and hypotonia. Pediatr Pulmonol. 2005; 40(3): 205 .

19. Bhattacharjee R, Kheirandish-Gozal L, Spruyt K, Mitchell RB, Promchiarak J, Simakajornboon N, et al. Adenotonsillectomy outcomes in treatment of obstructive sleep apnea in children: a multicenter retrospective study. Am J Respir Crit Care Med. 2010;182(5):676-83.

20. •• Friedman M, Wilson M, Lin H, Chang H. Updated systematic review of tonsillectomy and adenoidectomy for treatment of 
pediatric obstrutive sleep apnea/hypopnea syndrome. Otolaryngol, Head Neck Surg. 2009;140(6):800-808. Systematic review of response rates after adenotonsillectomy in children

21. Marcus CL. Pathophysiology of childhood obstructive sleep apnea: current concepts. Respir Physiol. 2000;119(2-3):143-54.

22. Breitzke SE, Katz ES, Roberson DW. Can history and physical examination reliably diagnose pediatric obstructive sleep apnea/ hypopnea syndrome? A systematic review of the literature. Otolaryngol Head Neck Surg. 2004;131:827.

23. Nolan J, Brietzke SE. Systematic review of pediatric tonsil size and polysomnogram-measured obstructive sleep apnea severity. Otolaryngol Head Neck Surg. 2011;144(6):844-50.

24. Li AM, Wong E, Kew J, Hui S, Fok TF. Use of tonsil size in the evaluation of obstructive sleep apnoea. Arch Dis Child. 2002; 87(2):156-9.

25. Jain A, Sahni JK. Polysomnographic studies in children undergoing adenoidectomy and/or tonsillectomy. J Laryngol Otol. 2002;116(9):711-5.

26. Lane F, Donnelly LF, Surdulescu V, Chini BA, Casper KA, Poe SA, et al. Upper airway motion depicted at cine MR imaging performed during sleep: comparison between young patients with and those without obstructive sleep apnea. Radiology. 2003;227: 239-45.

27. Shott SR, Donnelly LF. Cine magnetic resonance imaging: evaluation of persistent airway obstruction after tonsil and adenoidectomy in children with Down syndrome. Laryngoscope. 2004;114(10):1724-9.

28. Guimaraes C, Donnelly LF, Shott SR, Amin RS, Kalra M. Relative rather than absolute macroglossia in patients with Down syndrome: implications for treatment of obstructive sleep apnea. Pediatr Radiol. 2008;38:1062-7.

29. Schaaf WE, Wootten CT, Donnelly LF, Ying J, Shott SR. Findings on MR sleep studies as biomarkers to predict outcome of genioglossus advancement in the treatment of obstructive sleep apnea in children and young adults. Am J Roentgenol. 2010; 194(5): 120 .

30. Rizzi M, Onorato J, Andreoli A, Colombo S, Pecis M, Marchisio $P$, et al. Nasal resistances are useful in identifying children with severe obstructive sleep apnea before polysomnography. Int J Pediatr Otorhinolaryngol. 2002;65(1):7-13.

31. Monahan KJ, Larkin EK, Rosen CL, Graham G, Redline S. Utility of non-invasive pharyngometry in epidemiologic studies of childhood sleep-disordered breathing. Am J Respir Crit Care Med. 2002;165(11):1499-503.

32. Hessel NS, de Vries N. Diagnostic work-up of socially unacceptable snoring. Eur Arch Otorhinolaryngol. 2002;259(3):158-61.

33. Sadaoka T, Kakitsuba N, Fujiwara Y, Kanai R, Takahashi H. The value of sleep nasendoscopy in the evaluation of patients with suspected sleep-related breathing disorders. Clin Otolaryngol Allied Sci. 1996;21(6):485-9.

34. Pringle MB, Croft CB. A grading system for patients with obstructive sleep apnoea-based on sleep nasendoscopy. Clin Otolaryngol Allied Sci. 1993;18(6):480-4.

35. • Rodriguez-Bruno K, Goldberg AN, McCulloch C, Kezirian EJ. Test-retest reliability of drug-induced sleep endoscopy. Otolaryngol Head Neck Surg. 2009;140:646-651. Rare work to validate sleep endoscopy

36. •• Kezirian EJ, White DP, Malhotra A, McCulloch C, Goldberg A. Interrater reliability of drug induced sleep endoscopy. Arch Otolaryngol Head Neck Surg. 2010;136(4):393. Rare work to validate sleep endoscopy

37. - Sher A, Shprintzen RJ, Thorpy MJ. Endoscopic observations of obstructive sleep apnea in children with anomalous upper airways: predictive and therapeutic value. Int J Pediatr Otorhinolaryngol 1986;11(2):135-146. Essential reading for its place in assessing upper airway endoscopically in general
38. Croft CB, Thomson HG, Samuels MP, Southall DP. Endoscopic evaluation and treatment of sleep associated upper airway obstruction in infants and young children. Clin Otolaryngol Allied Sci. 1990;15(3):209-16.

39. Borowiecki B, Pollak CP, Weitzman ED, Rakoff S, Imperato J. Fiberoptic study of the pharyngeal airway during sleep in patients with hypersomnia, obstructive sleep apnea syndrome. Laryngoscope. 1978;88:1310-3.

40. Contencin P, Nottet JB, Yacoubian K, Soussi T, Nivoche Y, Narcy P. Pharyngolaryngeal fibroscopy under general anesthesia in children: technique and indications in sleep apnea and hypopnea. Ann Otolaryngol Chir Cervicofac. 1991;108(7):373-7.

41. • Myatt HM, Beckenham EJ. The use of diagnostic sleep nasendoscopy in the management of children with complex upper airway obstruction. Clin Otolaryngol Allied Sci. 2000;25(3):200. Commendable attempt at a structured reporting of endoscopy

42. • Thevasagayam M, Rodger K, Cave D, Witmans M, El-Hakim H. Prevalence of laryngomalacia in children presenting with sleepdisordered breathing. Laryngoscope 2010;120(8):1662-1666. A documentation of the epidemiology of laryngomalacia among patients with snoring/sleep disordered breathing

43. •• Fung E, Witmans M, Ghosh M, Cave D, El-Hakim H. Upper airway findings in children with Down syndrome on sleep nasopharyngoscopy: case-control study. J Otolaryngol Head Neck Surg. 2012;41(2):138-144. The proposed characterization of airway findings in terms of obstruction, collapse or a mixture is unique

44. - Truong MT, Woo VG, Koltai PJ. Sleep endoscopy as a diagnostic tool in pediatric obstructive sleep apnea. Int J Pediatr Otorhinolaryngol. 2012;76(5):722-727. Despite absence of structured algorithm the findings of explanatory airway pathology other than tonsil and adenoid enlargement is significant

45. Durr ML, Meyer AK, Kezirian EJ, Rosbe KW. Drug-induced sleep endoscopy in persistent pediatric sleep-disordered breathing after adenotonsillectomy. Arch Otolaryngol Head Neck Surg. 2012;138(7):638-64.

46. Kezirian EJ, Hohenhorst W, de Vries N. Drug-induced sleep endoscopy: the VOTE classification. Eur Arch Otorhinolaryngol. 2011;268(8):1233-6.

47. - Rabelo FAW, Braga A, Kupper DS, De Oliveira JAA, Lopes FM, de Lima Mattos,Pedro Luiz Vaz, et al. Propofol-induced sleep: polysomnographic evaluation of patients with obstructive sleep apnea and controls. Otolaryngol Head Neck Surg. 2010;142(2):218. Evidence of similarity of natural sleep to propofol induced sleep in generation of sleep disturbance

48. Eastwood PR, Szollosi I, Platt PR, Hillman DR. Comparison of upper airway collapse during general anaesthesia and sleep. Lancet. 2002;359(9313):1207-9.

49. Norton JR, Ward DS, Karan S, Voter WA, Palmer L, Varlese A, et al. Differences between midazolam and propofol sedation on upper airway collapsibility using dynamic negative airway pressure. Anesthesiology. 2006;104(6):1155-64.

50. • Hillman DR, Walsh JH, Maddison KJ, Platt PR, Kirkness JP, Noffsinger WJ, et al. Evolution of changes in upper airway compatibility during slow induction of anestheisa with propofol. Anesthesiology 2009;111(1):63-7. Evidence for a defined moment of physiologic change producing obstruction with propofol

51. Eastwood PR, Platt PR, Shepherd K, Maddison K, Hillman DR. Collapsibility of the upper airway at different concentrations of propofol anesthesia. Anesthesiology. 2005;103(3):470-7.

52. Eikermann M, Grosse-Sundrup M, Zaremba S, Henry ME, Bittner EA, Hoffmann U, et al. Ketamine activates breathing and abolishes the coupling between loss of consciousness and upper airway dilator muscle dysfunction. Anesthesiology. 2012;116(1):35-46.

53. Crawford MW, Arrica M, Macgowan CK, Yoo S. Extent and localization of changes in upper airway caliber with varying 
concentrations of sevoflurane in children. Anesthesiology. 2006; 105(6):1147-52.

54. Litman RS, McDonough JM, Marcus CL, Schwartz AR, Ward DS. Upper airway collapsibility in anesthetized children. Anesth Analg. 2006;102(3):750-4.

55. Mahmoud M, Gunter J, Donnelly LF, Wang Y, Nick TG, Sadhasivam S. A comparison of dexmedetomidine with propofol for magnetic resonance imaging sleep studies in children. Anesth Analg. 2009;109:745-53.

56. Chan DK, Truong MT, Koltai PJ. Supraglottoplasty for occult laryngomalacia to improve obstructive sleep apnea syndrome. Arch Otolaryngol Head Neck Surg. 2012;138(1):50-4.
57. Lin AC, Koltai PJ. Persistent pediatric obstructive sleep apnea and lingual tonsillectomy. Otolaryngol Head Neck Surg. 2009; 141(1):81-5.

58. Fung E, Cave D, Witmans M, Gan K, El-Hakim H. Postoperative respiratory complications and recovery in obese children following adenotonsillectomy for sleep-disordered breathing: a case-control study. Otolaryngol Head Neck Surg. 2010;142(6):898-905.

59. • Koutsourelakis I, Saffirudin F, Ravesloot M, Zakynthinos S, de Vries N. Surgery for obstructive sleep apnea: sleep endoscopy determinants of outcome. Laryngoscope 2012;122(11):2587-91. Attempt at predicting outcome of treatment from findings of endoscopy 